\title{
Cardiorespiratory response during combined training with breathing resistance and sustained physical exertion: a pilot study
}

Satoshi Kido ${ }^{1,2 *}$, Wenwei Yu ${ }^{3}$, Yasuhiro Nakajima ${ }^{4}$, Toshiaki Tanaka ${ }^{5,6}$, Tomoya Miyasaka ${ }^{6}$, Satoshi Shirogane ${ }^{7}$ Hiroshi Maruoka $^{1}$ and Kiyomi Takayanagi $^{1}$

${ }^{1}$ Department of Physical Therapy, Faculty of Health and Social Services, Saitama Prefectural University, Japan

${ }^{2}$ Department of Medical System Engineering, Graduate School of Engineering, Chiba University, Japan

${ }^{3}$ Center for Frontier Medical Engineering, Chiba University, Japan

${ }^{4}$ Industrial Research Institute, Hokkaido Research Organization, Japan

${ }^{5}$ Japan Institute of Gerontology, The University of Tokyo, Japan

${ }^{6}$ Department of Physical Therapy, Faculty of Health Sciences, Hokkaido University of Science, Japan

${ }^{7}$ Department of Assistive Technology, National Rehabilitation Center for Persons with Disabilities, Japan

\begin{abstract}
Purpose: In this pilot study, the cardiorespiratory response including respiratory muscle activity during combined training with breathing resistance and sustained physical exertion was determined in healthy adult males to evaluate the feasibility and safety of this novel training.

Methods: Eight healthy young males (mean age $=25.4$ years) randomly performed combined training with breathing resistance and sustained physical exertion and only sustained physical exertion training.

Heart rate, blood pressure, arterial oxygen saturation, respiratory parameters, and electromyography were obtained during both trainings.

Results: During combined training with breathing resistance and sustained physical exertion, respiratory rate and minute ventilation were significantly lower, whereas tidal volume and total respiratory time were significantly higher compared to only sustained physical exertion training. During combined training with breathing resistance and sustained physical exertion, the integrated electromyography recordings for the vastus medialis muscle were significantly lower, and integrated electromyography for the sternomastoid muscle was significantly higher compared to only sustained physical exertion training.

Conclusions: Results showed i) a change to a deeper and slower respiratory pattern, and ii) higher inspiratory muscle activity during combined training with breathing resistance and sustained physical exertion compared to only sustained physical exertion training in healthy young adult males. These features during combined training with breathing resistance and sustained physical exertion may influence the improvement in cardiorespiratory endurance and respiratory muscle function due to long-term this combined training.
\end{abstract}

\section{Introduction}

A number of studies have reported an association between cardiorespiratory endurance and cardiovascular disease morbidity and mortality rates $[1,2]$, implying that increased cardiorespiratory endurance may also be beneficial in promoting good health. Therefore, many different methods of training have been recommended, and the effectiveness of these methods has been examined. Rhythmic physical exercise such as running or cycling leads to improved maximum oxygen uptake $\left(\mathrm{VO}_{2 \max }\right)$ and ventilatory threshold (VT) [3]. The $\mathrm{VO}_{2 \max }$ and VT depend on respiratory function in addition to circulatory and muscular function [4]. However, although training such as running and cycling improves circulatory and muscular functions, the respiratory load may not be sufficiently large to improve $\mathrm{VO}_{2 \max }$ and VT. Therefore, respiratory muscle training (RMT) methods have been developed for use in both healthy $[5,6]$ and patient populations $[7,8]$ that improve cardiorespiratory endurance by improving respiratory muscle function. However, as each of these training methods consisted of respiratory loading in a sitting or standing position, it was not possible to simultaneously perform physical training with respiratory loading. For this reason, physical exercise needed to be performed separately, additional to RMT during exercise and rehabilitation sessions. In addition, most of the conventional RMT methods consisted of applying a resistive load on either inhalation or exhalation. Nevertheless, devices that applied resistive load during both inhalation and exhalation were rare. The research team hypothesized that effective benefits of the training could be gained if physical training and respiratory loading were to be performed simultaneously. In other words, it was predicted that respiratory function and cardiorespiratory endurance could

Correspondence to: Satoshi Kido, PT, M. S., Department of Physical Therapy, Faculty of Health and Social Services, Saitama Prefectural University, 820 SanNomiya Koshigaya-shi, Saitama, 343-8540, Japan, Tel \& Fax: +81-48-973-4173; E-mail: kido-satoshi@spu.ac.jp

Key words: respiratory muscles, breathing exercises, anaerobic threshold

Received: March 04, 2017; Accepted: April 07, 2017; Published: April 10, 2017 
improve by increasing inspiratory and expiratory muscle activity with resistive loading on both inhalation and exhalation. Based on this hypothesis, a 6-week combined training with breathing resistance and sustained physical exertionwas performed using a mask-type device that created inspiratory and expiratory resistance while only allowing inhalation through the nose and exhalation through the mouth. A comparison between the results of the 6-week combined training with breathing resistance and sustained physical exertion and a similar intensity physical training without respiratory load showed significant improvement in maximal voluntary ventilation and VT [9]. Compared to the respiratory training methods noted above, combined training with breathing resistance and sustained physical exertion has the following distinctions. First, as this training uses a mask-type device, it can be conducted in conjunction with physical exercise. This leads to a reduction in training time, which is an advantage. Also, the ventilator response during training may increase tidal volume (Tv) and decrease respiratory rate (RR) compared to physical exercise alone. Second, a rise in blood pressure and a drop in partial pressure of arterial oxygen may occur as a result of restricted ventilation from exercising with a respiratory load. Third, this combined training can place a load on both inspiratory and expiratory muscles. This in turn increases muscle activity of both the inspiratory and expiratory muscles and may be beneficial to respiratory function and improve cardiorespiratory endurance. However, little is known about the activity of the respiratory muscles and cardiorespiratory response during combined training with breathing resistance and sustained physical exertion.

A previous study aimed at proving the efficacy of combined training with breathing resistance and sustained physical exertion [9]; however the underlying mechanism of how this combined training improves the respiratory function has not been revealed.

Studying the physical responses to this novel combined training with breathing resistance and sustained physical exertion training method will: i) provide more insight into the characteristics of the training and be beneficial for enhancing safety considerations during training; ii) produce basic data for identifying the mechanism of improving cardiorespiratory endurance and respiratory muscle function; and iii) provide information for improvement of intervention methods, selection of suitable participants, optimization of degree of intervention, and investigation of the advantages and disadvantages of combining it with other methods. Therefore it was determined that the cardiorespiratory response including respiratory muscle activity during combined training with breathing resistance and sustained physical exertion in healthy adult males to evaluate the feasibility and safety of this training. Collecting basic information is important for further understanding of the physical response during combined training with breathing resistance and sustained physical exertion and for ensuring safety and the desired effects.

\section{Methods}

\section{Participants and setting}

For this pilot study a convenience sample was sought. Volunteer health males were recruited. Inclusion criteria included that the subject was not a preferential mouth breather, or did not had sinus issues limiting nasal inspiration. Individuals with a past history of respiratory and cardiovascular illnesses and recent injuries or orthopaedic / musculoskeletal limitations were excluded. The study was conducted at Saitama Prefectural University in Japan. Participants randomly shifted between combined training with breathing resistance and sustained physical exertion and only sustained physical exertion trainingusing simple randomization. The combined training with breathing resistance and sustained physical exertion and only sustained physical exertion training were conducted on the same day, with a 30-minute or more break in between. After it was confirmed that heart rate (HR) and $\mathrm{RR}$ returned to resting baseline levels, the subjects started the second training. Participants did not receive any financial reimbursement for participating. The study was approved by the Ethics Committee of Saitama Prefectural University (no. 23024), and the individuals consented to participate after receiving a written explanation of the details of the study.

\section{Exercise protocol}

Subjects followed a common exercise load protocol for both only sustained physical exertion training and combined training with breathing resistance and sustained physical exertion performed on a cycle ergometer (Ergometer 232C, Combi). Resting HR was confirmed after a few minutes of rest, and a 3-minute warm-up was initiated at 50 watts pedal power. After the warm-up, incremental loaded exercise (20 watt $/ \mathrm{min})$ was performed until a $75 \%$ maximal $\mathrm{HR}\left(\mathrm{HR}_{\max }\right)$ was reached. After reaching $75 \% \mathrm{HR}_{\max }$, participants continued to exercise for eight minutes with adjustment of the load every 30 seconds to maintain this HR. Pedal power was maintained at 50 watts for three minutes before discontinuing the exercise. Pedal rotation speed was $60 \mathrm{rpm}$. Calculation of $75 \% \mathrm{HR}_{\max }$ during exercise was calculated according to the formula:

$$
75 \% \mathrm{HR}_{\max }=(220-\text { age }(\text { years })) \times 75 \%
$$

When performing only sustained physical exertion training and combined training with breathing resistance and sustained physical exertion, blood pressure, arterial oxygen saturation $\left(\mathrm{SpO}_{2}\right)$, and subjective exercise intensity were measured every minute from start to finish. Ventilation parameters and surface electromyogram (EMG) were measured for 1 minute (arbitrary) between 5 and 8 minutes to measure the interval after reaching $75 \% \mathrm{HR}_{\max }$.

During the combined training with breathing resistance and sustained physical exertion, the subjects wore a ReBNA (Patent Works Inc) (Figure 1), which is a mask-type device with valves arranged such that inhalation is only through the nose and exhalation only through the mouth. Ventilation through two inspiratory valves and two expiratory valves produces respiratory load.

\section{Measurement}

\section{Hemodynamics and rate of perceived exertion}

The following were measured during the load exercises: HR (Multitelemeter, Nihon Kohden); blood pressure (STBP-780, Colin); $\mathrm{SpO}_{2}$

\section{Outside of the ReBNA Inside of the ReBNA}

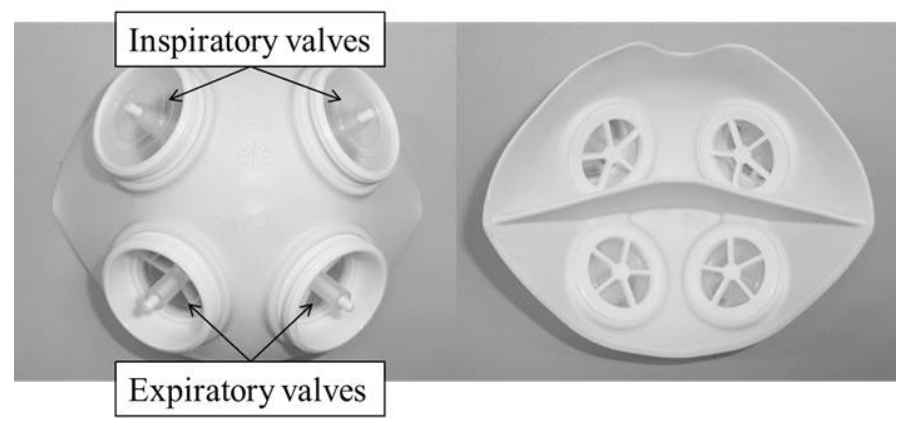

Figure 1. Appearance of the ReBNA. The mask has separate spaces inside for the nose and mouth. 
(Pulse Oximeter SAT-2200, Nihon Kohden); subjective fatigue of respiration and lower extremities (Borg scale [10]).

\section{Respiratory parameters}

The inhalation port of the facemask (Face mask series 7910, Hans Rudolph Inc.) used for measuring ventilation was opened, and the port was connected to a MEMS flow sensor D6F-50A6-000 (OMRON) through the split tubing from the inhalation port. During combined training with breathing resistance and sustained physical exertion, the facemask was worn so that it covered the entire ReBNA. The inspiratory flow data were transferred to a computer from the analog to digital interface (AO16CH, Applied Office). Exercise was performed on 1 day, and sensor calibrations (the flow rate of pumping out $3 \mathrm{~L}$ from the syringe) were performed five times on 3 days for different subjects. After offsetting the measured voltage to zero, integration was performed. The voltage-flow rate coefficient was determined so that the obtained value became $3 \mathrm{~L}$, and the average of 15 batches was used. Measurement accuracy (precision) was $\pm \mathrm{a}=1.75 \%$. DASYLab-V9.00.02 (National Instruments) was used for conversion of the measured voltage data of the flow processor during inhalation and for calculating each parameter. The parameters were $\mathrm{RR}, \mathrm{TV}$, minute volume of ventilation (VE), total respiratory cycle time for complete respiratory cycle (T tot), inspiration time (Ti), and Ti/T tot. The Tv and VE of combined training with breathing resistance and sustained physical exertion were calculated using the correction coefficient obtained from the results of the maximal inspiratory capacity at rest measured three times before the test for each subject with the ReBNA.

\section{Surface EMG}

Surface EMG electrodes were placed as follows: i) vastus medialis muscle [midpoint placed $1 / 5$ of the way from the knee joint on the knee joint-to-pelvic anterior superior iliac spine line as the dominant motion muscle for the bicycle ergometer; ii) sternomastoid muscle (an auxiliary muscle) [midpoint placed $1 / 3$ of the way from the mastoid process on the mastoid process-to-jugular notch line] as the inspiratory muscle; and iii) external oblique muscle (a forced expiratory muscle) [midpoint of the lower margin of the $10^{\text {th }}$ rib] and abdominal rectus muscle [midpoint placed two fingerbreadths outside and two fingerbreadths under the linea alba of the ensiform cartilage] as expiratory muscles. The distance between electrodes was half a fingerbreadth. Dispo Electrode F (Nihon Kohden) for electrocardiogram monitors was placed parallel to the muscle fiber. The derived EMG was amplified with EMG Master 4ch EMG Amp (Mediarea Support Business Union) and transferred to a computer after analog-digital conversion with a sampling frequency of $1 \mathrm{kHz}$. All myoelectric signals for 1 minute were full-wave rectified, and the integration value was calculated after HICUT: $200 \mathrm{~Hz}$ and LOCUT:5 Hz were obtained. The integrated EMG (iEMG) was calculated for each muscle, and mean values of the subjects were obtained.

\section{Data analysis}

Statistical analysis was performed using SPSS Statistics Version 19 (IBM). Measured data for only sustained physical exertion training and combined training with breathing resistance and sustained physical exertion were tested for normality using the Shapiro-Wilk test. The Student's t-test was used when the parameters followed a normal distribution. The Wilcoxon signed-rank test was used in cases where normality was not valid. The statistical significance level was set at 0.05 .

\section{Results}

Eight health young male volunteers (age $25.4 \pm 5.3$ years, height $172.1 \pm 4.9 \mathrm{~cm}$, weight $64.5 \pm 7.3 \mathrm{~kg}$ ) participated. All participants completed the study. The anthropometric data for all participants are presented in Table 1.

Effect of combined training with breathing resistance and sustained physical exertion on hemodynamics and the rate of perceived exertion

Comparison of cardiorespiratory parameters, subjective exercise intensity and load of bicycle ergometer during training are presented in Table 2.No statistically significant difference between only sustained physical exertion training and combined training with breathing resistance and sustained physical exertion was found in maximum systolic blood pressure (SBPmax), maximum diastolic blood pressure (DBPmax), dyspnea Borg score [10], lower extremity fatigue Borg score, or work rate.

\section{Effect of combined training with breathing resistance and} sustained physical exertion on respiratory parameters

Respiratory parameters during training are presented in Table 3.

Table 1. Anthropometric data for all participants

\begin{tabular}{|c|c|c|c|c|c|}
\hline & Height (cm) & Weight (kg) & Age (years) & $\begin{array}{c}\text { Body mass } \\
\text { index }\end{array}$ & $\begin{array}{c}\text { Exercise } \\
\text { frequency } \\
\text { (days/week) }\end{array}$ \\
\hline $\begin{array}{c}\text { Mean of all } \\
\text { subjects }\end{array}$ & 172.1 & 64.5 & 25.4 & 21.8 & 1.1 \\
\hline $\begin{array}{c}\text { SD of all } \\
\text { subjects }\end{array}$ & 4.9 & 7.3 & 5.3 & 2.9 & 2.5 \\
\hline
\end{tabular}

Exercise frequency was the days carrying out physical exercise one hour or more per week.

Table 2. Comparison of cardiorespiratory parameters, subjective exercise intensity, and load of bicycle ergometer during training

\begin{tabular}{|c|c|c|c|}
\hline Parameters & $\begin{array}{c}\text { Only sustained physical } \\
\text { exertion training }\end{array}$ & Combined training & P value \\
\hline SpO $_{2}$ min, \% & $97.0 \pm 0.5(96.0-98.0)$ & $95.6 \pm 0.6(94.5-96.8)$ & 0.008 \\
\hline SBPmax, mmHg & $179.0(166.8-192.0)$ & $194.0(185.3-197.3)$ & 0.540 \\
\hline DBPmax, mmHg & $89.5 \pm 6.1(77.6-101.4)$ & $103.6 \pm 9.3(85.4-121.8)$ & 0.259 \\
\hline Dyspnea Borg Score & $15.0(13.0-16.0)$ & $15.5(13.8-17.3)$ & 0.064 \\
\hline $\begin{array}{c}\text { Lower Extremity } \\
\text { Fatigue Borg score }\end{array}$ & $15.0(12.3-17.0)$ & $14.0(11.5-15.5)$ & 0.186 \\
\hline Work Rate, W & $100(100.0-107.5)$ & $105(98.5-117.5)$ & 0.749 \\
\hline
\end{tabular}

Abbreviations: $\mathrm{SpO}_{2}$ min, minimal arterial oxygen saturation; SBPmax, maximum systolic blood pressure; DBPmax, maximum diastolic blood pressure.

Variables that were normally distributed are presented as the mean \pm SE $(95 \%$ confidence interval) and were tested with the Student's t-test. Skewed variables displayed in italics_are presented as the median (interquartile range) and were tested with the Wilcoxon signedrank test.

Table 3. Respiratory parameters during training

\begin{tabular}{|c|c|c|c|}
\hline Parameters & $\begin{array}{c}\text { Only sustained physical exertion } \\
\text { training }\end{array}$ & Combined training & P value \\
\hline $\mathbf{R R}, \mathbf{b p m}$ & $17.0(15.0-21.5)$ & $15.0(13.5-15.3)$ & 0.018 \\
\hline $\mathbf{T v}, \mathbf{L}$ & $2.2 \pm 0.1(1.9-2.4)$ & $2.7 \pm 0.2(2.3-3.0)$ & 0.001 \\
\hline $\mathbf{V E}, \mathbf{L} / \mathbf{m i n}$ & $40.3 \pm 1.9(36.6-44.0)$ & $37.1 \pm 1.9(33.5-40.8)$ & 0.029 \\
\hline $\mathbf{T i}, \mathbf{s}$ & $1.8 \pm 0.1(1.6-2.1)$ & $1.9 \pm 0.1(1.8-2.1)$ & 0.257 \\
\hline $\mathbf{T}$ tot, $\mathbf{s}$ & $3.3 \pm 0.3(2.7-3.8)$ & $4.1 \pm 0.4(3.4-4.7)$ & 0.018 \\
\hline $\mathbf{T i} / \mathbf{T}$ tot & $0.6 \pm 0.0(0.5-0.6)$ & $0.5 \pm 0.0(0.4-0.5)$ & 0.006 \\
\hline
\end{tabular}

Abbreviations: RR, respiratory rate; $\mathrm{Tv}$, tidal volume; $\mathrm{VE}$, minute volume of ventilation; $\mathrm{Ti}$, inspiration time; $\mathrm{T}$ tot, total respiratory cycle time for complete respiratory cycle. Variables that were normally distributed are presented as mean \pm SE $(95 \%$ confidence interval) and were tested with the Student's t-test. Skewed variables displayed in italics are presented as the median (interquartile range) and were tested with the Wilcoxon signedrank test. 


\section{Effect of combined training with breathing resistance and sustained physical exertion on surface EMG}

Comparison of EMG during training are presented in Table 4.As seen on the EMG of a typical subject during measurement, the sternomastoid muscle activity during inspiration, especially in the later part of inspiration, increased during combined training with breathing resistance and sustained physical exertion compared to only sustained physical exertion training (Figure 2).

\section{Discussion}

A comparison between combined training with breathing resistance and sustained physical exertion and only sustained physical exertion training of equivalent intensity in healthy young volunteers showed i) deep and slow respiratory patterns; and ii) increased activity of the accessory inspiratory muscles. A comparison between combined training with breathing resistance and sustained physical exertion and only sustained physical exertion training of equivalent intensity in healthy young volunteers demonstrated that $\mathrm{SpO}_{2}$ was lower during training, confirming the hypothesis.

In combined training with breathing resistance and sustained physical exertion, the mask device worn during physical exercise imposes a resistive load on the respiratory system. A drop in $\mathrm{SpO}_{2}$ may have been triggered by the limited oxygen intake due to respiration and/ or increased oxygen consumption by the respiratory muscles. However, the effects of low arterial oxygen to the body appear to be limited, considering the fact that the range of exercise intensity in this study yielded an average $\mathrm{SpO}_{2}$ min of $95.6 \%$ during combined training with breathing resistance and sustained physical exertion, which exceeded the discontinuance criteria of $90 \%$ for stable patients with respiratory disorders during exercise therapy [11]. That said, as hypoxemia could readily occur during training, extra precautions should be taken when combined training with breathing resistance and sustained physical exertion is used in participants with compromised respiratory functions such as in the elderly and patients with respiratory disorders. However, this recommendation is until research is done to investigate with these cohorts. Blood pressure control during training is important for safety reasons, as an excessive rise in blood pressure during exercise therapy could burden the cardiovascular system, potentially triggering strokes or cardiovascular events. According to current study results, there was no significant difference in $\mathrm{SBP}_{\text {max }}$ or $\mathrm{DBP}_{\text {max }}$ between only sustained physical exertion training and combined training with breathing resistance and sustained physical exertion. Therefore, compared to conventional training methods, combined training with breathing resistance and sustained physical exertion can be conducted without having to pay special attention to the rise in blood pressure at exercise intensities of up to $75 \% \mathrm{HR}_{\max }$ in healthy young individuals.

The results showed decreased RR, increased TV, and a deep and slow respiratory pattern during combined training with breathing resistance and sustained physical exertion compared to only sustained physical exertion training. Deep and slow respiration during endurance training leads to a decrease in the ratio of dead space to Tv, thereby improving ventilation efficiency. Compared to only sustained physical exertion training, there was a decrease in VE during combined training with breathing resistance and sustained physical exertion, and this may have been due to the improvement in the alveolar ventilation ratio during combined training with breathing resistance and sustained physical exertion. The results showed no significant difference in Ti between only sustained physical exertion training and combined training with breathing resistance and sustained physical exertion; however, the significantly longer $\mathrm{T}$ tot indicated that expiratory time was increased during combined training with breathing resistance and sustained physical exertion.

The recent study of Hellyer et al. [12] reported the respiratory muscle activity during simultaneous stationary cycling and inspiratory muscle training. In their study, simultaneous stationary cycling with only inspiratory load did not significantly raise sternomastoid muscle potential than it without inspiratory load. However this present study demonstrated that the amount of activity of the inspiratory and skeletal muscles differed between combined training with breathing resistance and sustained physical exertion and no-respiratory load

Table 4. Comparison of EMG during training

\begin{tabular}{|c|c|c|c|}
\hline Muscle & $\begin{array}{c}\text { Only sustained physical } \\
\text { exertion training }\end{array}$ & Combined training & P value \\
\hline $\begin{array}{c}\text { Vastus Medialis } \\
\text { Muscle, } \mathrm{mV} \cdot \mathrm{s}\end{array}$ & $179.0(163.7-204.4)$ & $133.7(122.9-193.9)$ & 0.012 \\
\hline $\begin{array}{c}\text { Sternomastoid } \\
\text { Muscle, } \mathrm{mV} \cdot \mathrm{s}\end{array}$ & $26.4 \pm 4.4(17.7-35.0)$ & $65.8 \pm 11.5(43.2-88.4)$ & 0.014 \\
\hline $\begin{array}{c}\text { Abdominal Rectus } \\
\text { Muscle, } \mathrm{mV} \cdot \mathrm{s}\end{array}$ & $25.5 \pm 6.7(12.4-38.7)$ & $33.8 \pm 8.3(17.6-50.1)$ & 0.484 \\
\hline $\begin{array}{c}\text { External Oblique } \\
\text { Muscle, } \mathrm{mV} \cdot \mathrm{s}\end{array}$ & $29.5 \pm 8.8(12.3-16.7)$ & $36.5 \pm 9.5(17.8-55.1)$ & 0.380 \\
\hline
\end{tabular}

Variables that were normally distributed are presented as the mean $\pm \mathrm{SE}(95 \%$ confidence interval) and were tested with the Student's t-test. Skewed variables displayed in italics are presented as the median (interquartile range) and were tested with the Wilcoxon signedrank test.
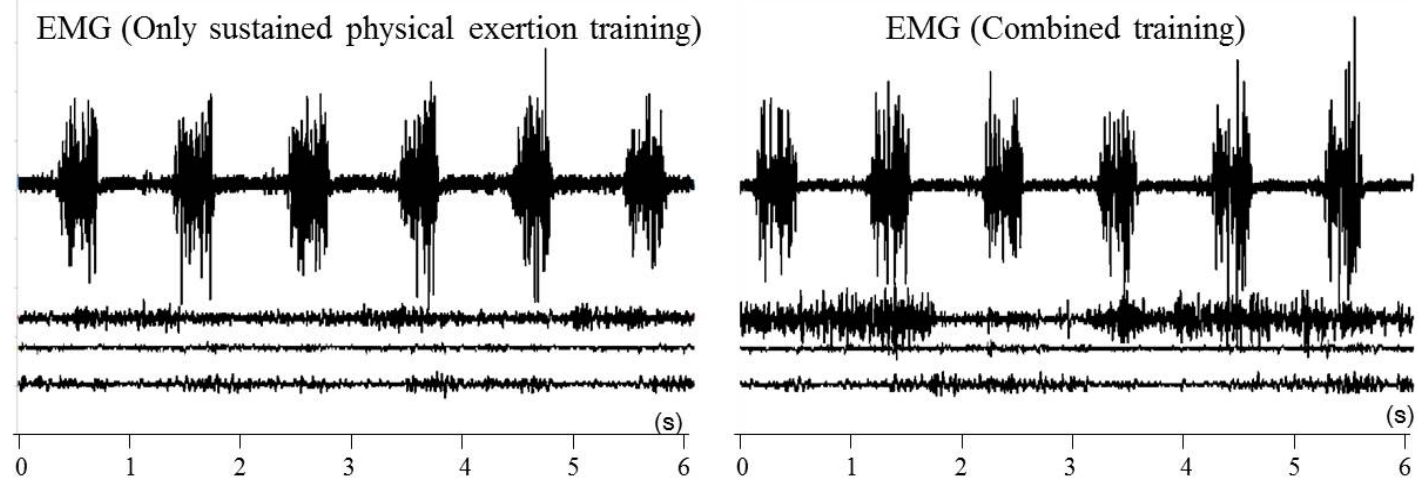

Left: During only sustained physical exertion training. Right: During combined training with breathing resistance and sustained physical exertion. From top to bottom: EMG of the vastusmedialis muscle, sternomastoid muscle, abdominal rectus muscle, and external oblique muscle.

Figure 2. A typical subject EMG 
training. It is a difference that combined training with breathing resistance and sustained physical exertion method have the expiratory load in addition to the inspiratory load compared with the method of Hellyer et al. [12]. Based upon the foregoing, it is considered that sternomastoid muscle activity is raised by expiratory load increasing in addition to inspiratory load in combined training with breathing resistance and sustained physical exertion. Compared to only sustained physical exertion training, the iEMGs of combined training with breathing resistance and sustained physical exertion were small for the vastus medialis muscle and large for the sternomastoid muscle. In other words, there was a decrease in vastus medialis muscle activity, which is the agonist muscle in pedaling action, along with increased activity of the sternomastoid muscle, which is involved in inspiration. The different load distributions on the various muscles during training provide fundamental data that physiologically describe the training effects. This information is useful to determine how one can effectively increase the benefits of training and safely conduct the exercises so that respiratory load and exercise intensity of combined training with breathing resistance and sustained physical exertion can be adapted and adjusted for use in the elderly. The active inspiration due to combined training with breathing resistance and sustained physical exertion showed that combined training with breathing resistance and sustained physical exertion may also be effective as a training method to improve inspiratory muscle function similar to training methods [6] that use mechanical loading. In the future, the establishment and evaluation of a procedure for measuring not only sternomastoid muscle activity as an accessory inspiratory muscle, but also muscle activity of the diaphragm and intercostal muscle, which contribute greatly as inspiratory muscles [13], will enable a more comprehensive evaluation of the effects of combined training with breathing resistance and sustained physical exertion on respiratory muscle activity. On the other hand, there was no significant change observed in the expiratory muscles such as the abdominal rectus and eternal oblique muscles, with combined training with breathing resistance and sustained physical exertion. Loading strength on expiration in this study may not have been sufficient for increasing the measured expiratory muscle activity. Wearing a mask-type device while exercising affected the respiratory rhythm, for instance by extending the expiratory time. Activity levels of muscles not measured in this study, such as the internal oblique and transverse abdominal muscles, may have increased.

\section{Study limitations}

This study was performed with healthy young individuals with no impairment in respiratory function. Therefore, the study results and physical response observed in this study may not apply to individuals with impaired respiratory or physical functions such as the elderly and patients with respiratory disorders.

However, the findings of the study are important for improvement of intervention methods, selection of suitable participants, optimization of degree of intervention, and investigation of the advantages and disadvantages of combining it with other methods.

\section{Conclusion}

This study results showed i) a change to a deeper and slower respiratory pattern; and ii) higher inspiratory muscle activity during combined training with breathing resistance and sustained physical exertion compared to only sustained physical exertion training among healthy young adult individuals. Changes in the respiratory pattern and $\mathrm{VE}$ of ventilation during combined training with breathing resistance and sustained physical exertion, and variations in muscle activities are new discoveries that will be valuable for increasing physiological understanding of the characteristics of combined training with breathing resistance and sustained physical exertion in order to establish this combined training as a safe and effective exercise therapy.

\section{Acknowledgment} device.

The authors thank Patent Works Inc. for providing the mask-type

This research was conducted in Saitama Prefectural University.

\section{Funding}

This work was supported in part by an FY research grant from Saitama Prefectural University and JSPS KAKENHI Grant Numbers JP26750192.

\section{Disclosure of interest}

No conflicts of interests were declared by the authors in relation to this article.

\section{References}

1. Erez A, Kivity S, Berkovitch A, Milwidsky A, Klempfner R, et al. (2015) The association between cardiorespiratory fitness and cardiovascular risk may be modulated by known cardiovascular risk factors. Am Heart J 169: 916-923. [Crossref]

2. Harber MP, Kaminsky LA, Arena R et al. (2017) Impact of cardiorespiratory fitness on allcause and disease-specific mortality: advances since 2009. Prog Cardiovasc Dis. [Crossref]

3. Thompson PD, Arena R, Riebe D, Pescatello LS (2013) ACSM's guidelines for exercise testing and prescription, ninth edition. New York: Lippincott Williams and Wilkins.

4. Wasserman K, Van Kessel AL, Burton GG (1967) Interaction of physiological mechanisms during exercise. $J$ Appl Physiol 22: 71-85.

5. McMahon ME, Boutellier U, Smith RM, Spengler CM (2002) Hyperpnea training attenuates peripheral chemosensitivity and improves cycling endurance. J Exp Biol 205: 3937-3943. [Crossref]

6. Enright SJ, Unnithan VB (2011) Effect of inspiratory muscle training intensities on pulmonary function and work capacity in people who are healthy: a randomized controlled trial. Phys Ther 91: 894-905. [Crossref]

7. Sturdy G, Hillman D, Green D, Jenkins S, Cecins N, et al. (2003) Feasibility of highintensity, interval-based respiratory muscle training in COPD. Chest 123: 142-150. [Crossref]

8. Ramirez-Sarmiento A, Orozco-Levi M, Guell R, Barreiro E, Hernandez N, et al. (2002) Inspiratory muscle training in patients with chronic obstructive pulmonary disease structural adaptation and physiologic outcomes. Am J Respir Crit Care Med 166: 14911497. [Crossref]

9. Kido S, Nakajima Y, Miyasaka T et al. (2013) Effects of combined training with breathing resistance and sustained physical exertion to improve endurance capacity and respiratory muscle function in healthy young adults. J Phys Ther Sci 25: 605-610. [Crossref]

10. Borg GA (1982) Psychophysical bases of perceived exertion. Med Sci Sports Exerc 14 377-381. [Crossref]

11. AACVPR (2011) Guidelines for Pulmonary Rehabilitation Programs, 4th Edition. Champaign: Human Kinetics.

12. Hellyer NJ, Folsom IA, Gaz DV et al. (2015) Respiratory muscle activity during simultaneous stationary cycling and inspiratory muscle training. $J$ Strength Cond Res 29: 3517-3522. [Crossref]

13. Mead J, Smith JC, SH L (1985) Volume displacements of the chest wall and their mechanical significance. In: Roussos C, Macklem PT (Eds.), The thorax: part A. New York: Dekker.

Copyright: (C2017 Kido S. This is an open-access article distributed under the terms of the Creative Commons Attribution License, which permits unrestricted use, distribution, and reproduction in any medium, provided the original author and source are credited. 